\title{
Charged Excitons in a Dilute 2D Electron Gas in a High Magnetic Field
}

\author{
Arkadiusz Wójs \\ Department of Physics, University of Tennessee, Knoxville, Tennessee 37996 \\ Institute of Physics, Wroclaw University of Technology, Wroclaw 50-370, Poland \\ John J. Quinn \\ Department of Physics, University of Tennessee, Knoxville, Tennessee 37996 \\ Pawel Hawrylak \\ Institute for Microstructural Sciences, National Research Council of Canada, Ottawa, Canada K1A OR6
}

\begin{abstract}
A theory of charged excitons $X^{-}$in a dilute $2 \mathrm{D}$ electron gas in a high magnetic field is presented. In contrast to previous calculations, three bound $X^{-}$states (one singlet and two triplets) are found in a narrow and symmetric GaAs quantum well. The singlet and a "bright" triplet are the two optically active states observed in experiments. The bright triplet has the binding energy of about $1 \mathrm{meV}$, smaller than the singlet and a "dark" triplet. The interaction of bound $X^{-}$'s with a dilute 2D electron gas is investigated using exact diagonalization techniques. It is found that the short-range character of the $e-X^{-}$interactions effectively isolates bound $X^{-}$states from a dilute $e-h$ plasma. This results in the insensitivity of the photoluminescence spectrum to the filling factor $\nu$, and an exponential decrease of the oscillator strength of the dark triplet $X^{-}$as a function of $\nu^{-1}$.

71.35.Ji, 71.35.Ee, 73.20.Dx
\end{abstract}

\section{INTRODUCTION}

The magneto-optical properties of quasi-twodimensional (2D) electron systems have been intensirely investigated experimentally 13 and theoretically. $14-30$ For a dilute electron gas, the photoluminescence (PL) spectrum is determined by a charged-exciton complex $X^{-}$and its interaction with remaining electrons. The $X^{-}$consists of two electrons and a valence hole and is similar to the hydrogen ion $\mathrm{H}^{-}$. Its existence ip bulk semiconductors was first predicted by Lampert, 11 but due to small binding energy it has not been observed experimentally. Stebe and Ainane 18 showed that the binding of the second electron to the exciton $X$ should be enhanced in 2D systems. Indeed, the $X^{-}$has been observef in semiconductor quantum wells (Ry) by Kheng et al. 1 and in many related experiments. 113

The experimental observation stimulated a number of theoretical works. 1727 It is now well established that the only bound $X^{-}$state at zero magnetic field is the singlet state $\left(X_{s}^{-}\right)$with the total electron spin $J_{e}=0$. Accordingly, the PL spectrum shows only two peaks, due to the $X$ and $X_{s}^{-}$recombination, split by the $X_{s}^{-}$binding energy $\Delta_{s}$. The situation is much more complicated in a magnetic field. In very high fields, MacDonald and Rezayi 14 showed that optically active magneto-excitons do not bind a second electron. They are effectively decoupled from the excess electrons due to the "hidden symmetry,"15 17 and the PL spectrum is that of a single exciton, irrespective of the number of electrons present.

It was therefore surprising when a bound $X^{-}$complex was discovered via numerical experiments in the lowest Landau level (LL) 20 The bound complex was a triplet $\left(X_{t}^{-}\right)$with finite total angular momentum and a macro- scopic degeneracy. It was later shown by Palacios et al.21 that an isolated $X_{t}^{-}$in the lowest LL has infinite radiative time $\tau_{t}$. Two independent symmetries must be broken to allow for the $X_{t}^{-}$recombination: the "hidden symmetry"14 16, 21 due to an equal strength of $e-e$ and $e-h$ interactions and the 2D geometrical (translational) symmetry $17,25,22$ resulting in the conservation of two angular momentum quantum numbers. The "hidden symmetry" can be broken by mixing of LL's, valence band mixing effects, and asymmetry of the QW. The translational symmetry can be broken by disorder. Therefore, the $X_{t}^{-}$recombination probability is determined by disorder and scattering by additional electrons, and is expected to disappear with increasing magnetic field. Also, crossing of the $X_{t}^{-}$and $X_{s}^{-}$PL peaks must occur at some value of the magnetic field, when $X_{t}^{-}$becomes the $X^{-}$ ground state. This hypothetical long-lived $X_{t}^{-}$ground state in high magnetic fields has recently received a lot of attention. Because the $X_{t}^{-}$complexpes carry a net charge and form LL's, they are expected2526 to form (together with remaining electrons) the multi-component incompressible fluid states with Laughlin-Halperin 33 . 34 (LH) correlations. Since an experimental realization of such states requires reaching the "hidden symmetry" regime (long-lived $X_{t}^{-}$ground state), an estimate of required magnetic fields is needed.

While variationalcalculations of hydrogen-like $X_{s}^{-}$appear satisfactory, 182223 an accurate description of $X_{t}^{-}$ at finite magnetic fields is extremely difficult. Although Whittaker and Shield 24 (WS) predicted a transition to the $X_{t}^{-}$ground state in a GaAs/AlGaAs QW of width $w=10 \mathrm{~nm}$ at the magnetic field of $B \approx 30 \mathrm{~T}$, the experimental data for $B \leq 10 \mathrm{~T}$ that was available at the time 3 目 could not verify their result. A negative answer 
came recently from Hayne et al., whose PL measurements in magnetic fields up to $50 \mathrm{~T}$ seemingly precluded such transition. In their spectra, $X_{s}^{-}$remained the ground state up to $50 \mathrm{~T}$, and an extrapolation to higher fields ruled out the singlet-triplet crossing at any near values. Moreover, in clear disagreement with Ref. 21, strong $X_{t}^{-} \mathrm{PL}$ was detected, whose intensity increased with increasing the magnetic field, and which at $13.5 \mathrm{~T}$ exceeded that of the $X_{s}^{-}$. Results of Hayne et al. not only disagreed with the model of WS, but also suggested that a picture 21.25.26 of long-lived $X_{t}^{-}$'s forming the low energy states of an $e-h$ plasma, worked out for a strictly $2 \mathrm{D}$ system $(w=0)$ in the lowest LL, might be totally inadequate to realistic GaAs systems. This suspicion was further reinforced by the unexplained lack of the sensitivity of PL to the filling factor of the electron gas. The source of disagreement might be either in the description of bound $X^{-}$states or in the description of its interaction with excess electrons.

In this paper we address both issues. We report on detailed numerical calculations of the energy and PL spectra of $e^{-} h_{-}$systems at high magnetic fields. Using Lanczos-based 35 methods we were able to include in our model the effects of Coulomb interaction, LL mixing, finite QW width, and realistic Zeeman and cyclotron splittings. Our calculations predict the existence of a new, optically active bound state $X_{t b}^{-}$of the triplet charged exciton. The identification of this new state as the triplet $X^{-}$ state observed in PL explains the puzzling qualitative disagreement between earlier theory and experiments. The "bright" $X_{t b}^{-}$state is distinguished from the "dark" state $X_{t d}^{-}$found in earlier calculations20.21.24, 26, which is the lowest-energy triplet $X^{-}$state at high magnetic field but remains undetected in PL experiments (however, see also Ref. 36). Energies and oscillator strengths of all bound complexes: $X, X_{s}^{-}, X_{t b}^{-}$, and $X_{t d}^{-}$, are calculated as a function of the magnetic field and $\mathrm{QW}$ width. The transition to the $X_{t d}^{-}$ground state at $B \approx 30 \mathrm{~T}$ is confirmed.

The interaction of $X^{-}$'s with additional electrons is also studied. Because this interaction has short range, it effectively isolates the bound $X^{-}$states from remaining electrons and only weakly affects PL from dilute systems, as observed by Priest et al 1 In particular, collisions of $X_{t d}^{-}$with surrounding electron gas at filling factors $\nu<$ $1 / 5$ do not significantly enhance its oscillator strength. This explains why this state is not observed in PL.

\section{MODEL}

In order to preserve the 2D translational symmetry of an infinite QW in a finite-size calculation, electron-and holes are put on a surface of the Haldane sphere 3738 of radius $R$. The reason to choose the spherical geometry for calculations is strictly technical and of no physical consequence for our results. Because of the finite LL degeneracy, the numerical calculations on a sphere can be done without cutting off the Hilbert space and thus without breaking the $2 \mathrm{D}$ translational symmetry. This allows exact resolution of the two quantum numbers conserved due to this symmetry: the total $\left(\mathcal{L}_{\text {tot }}\right)$ and center-ofmass $\left(\mathcal{L}_{\mathrm{cm}}\right)$ angular momenta. Let us note that in earlier calculations, WS24 and Chapman et al.23 used planar geometry and hence could not resolve the $\mathcal{L}_{\mathrm{cm}}$ quantum number, which is essential to correctly identify the bound $X^{-}$states and to further accurately calculate their energy and PL. The exact mapping 39 between the $\mathcal{L}_{\text {tot }}$ and $\mathcal{L}_{\mathrm{cm}}$ quantum numbers on a plane and the 2D algebra of the total angular momentum on a sphere (and between the respective Hilbert eigensubspaces) allows conversion of the results from one geometry to the other. The price paid for closing the Hilbert space without breaking symmetries is the surface curvature which modifies interactions. However, if the correlations to be modeled have short range that can be described by a small characteristic length $\delta$, the effects of curvature are scaled by a small parameter $\delta / R$ and can be eliminated by extrapolating the results to $R \rightarrow \infty$. Therefore, despite all differences, the spherical geometry is equally well suited to modeling bound complexes as to the fractiopal quantum Hall systems (as originally used by Haldane 37 ).

The detailed description of the Haldane sphere model can be found e.g. in Refs. 37 and (since it is not essential for our results) it will not be repeated here. The magnetic field $B$ perpendicular to the surface of the sphere is due to a magnetic monopole placed in the center. The monopole strength $2 S$ is defined in the units of elementary flux $\phi_{0}=h c / e$, so that $4 \pi R^{2} B=2 S \phi_{0}$ and the magnetic length is $\lambda=R / \sqrt{S}$. The single-particle states are the eigenstates of angular momentum $l$ and its projection $m$ and are called monopole harmonics. The energies $\varepsilon$ fall into $(2 l+1)$-fold degenerate angular momentum shells separated by the cyclotron energy $\hbar \omega_{c}$. The $n$-th $(n \geq 0)$ shell (LL) has $l=S+n$ and thus $2 S$ is a measure of the system size through the LL degeneracy. Due to the spin degeneracy, each shell is further split by the Zeeman gap.

Our model applies to the narrow and symmetric QW's, and the calculations have been carried out for the GaAs/AlGaAs structures with the Al concentration of $x=0.33$ and the widths of $w=10 \mathrm{~nm}, 11.5 \mathrm{~nm}$, and $13 \mathrm{~nm}$. For such systems, only the lowest QW subband need be included and the cyclotron motion of both electrons and holes can be well described in the effectivemass approximation.24 For the holes, only the heavy-hole states are included, with the inter-subband coupling partially taken into account through the realistic dependence $\hbar \omega_{c h}(B)$, i.e. through the dependence of the effective inplane (cyclotron) mass $m_{h}^{*}$ on $B$ (after Cole et al.42).

Using a composite index $i=[n m \sigma]$ ( $\sigma$ is the spin projection), the $e-h$ Hamiltonian can be written as

$$
H=\sum_{i, \alpha} c_{i \alpha}^{\dagger} c_{i \alpha} \varepsilon_{i \alpha}+\sum_{i j k l, \alpha \beta} c_{i \alpha}^{\dagger} c_{j \beta}^{\dagger} c_{k \beta} c_{l \alpha} V_{i j k l}^{\alpha \beta},
$$


where $c_{i \alpha}^{\dagger}$ and $c_{i \alpha}$ create and annihilate particle $\alpha$ (e or $h)$ in state $i$, and $V_{i j k l}^{\alpha \beta}$ are the Coulomb matrix elements. At high magnetic fields, $w$ significantly exceeds $\lambda$ and it is essential to properly include the effects due to the finite QW width. Merely scaling all matrix elements $V_{i j k l}^{\alpha \beta}$ by a constant factor $\xi(w / \lambda)$ is not enough. Ideally, the $V_{i j k l}^{\alpha \beta}$ should be calculated for the actual 3D electron and hole wavefunctions.24 The "rod" geometry used by Chapman et al.23 might be a reasonable approximation (for the lowest QW subband), although using the same effective rod length for electrons and holes and its arbitrary scaling with $B$ leads to an incorrect $B$-dependence of obtained results. In this work we insist on using numerically correct values of $V_{i j k l}^{\alpha \beta}$ and calculate them in the following way. The actual density profile across the QW can be approximated by $\varrho(z) \propto \cos ^{2}\left(\pi z / w^{*}\right)$, i.e. by replacing the actual QW by a wider one, with an infinite potential step at the interface. This defines the effective widths of electron and hole layers, $w_{e}^{*}$ and $w_{h}^{*}$. For $w \sim 10 \mathrm{~nm}$, we obtain $w^{*} \equiv\left(w_{e}^{*}+w_{h}^{*}\right) / 2=w+2.5 \mathrm{~nm}$. We have checked that the effective $2 \mathrm{D}$ interaction in a quasi-2D layer,

$$
V(r)=\int d z \int d z^{\prime} \frac{\varrho(z) \varrho\left(z^{\prime}\right)}{\sqrt{r^{2}+\left(z-z^{\prime}\right)^{2}}}
$$

can be well approximated 43 by $V_{d}(r)=1 / \sqrt{r^{2}+d^{2}}$ if an effective separation across the QW is taken as $d=w^{*} / 5$. For a given $d / \lambda$, matrix elements of $V_{d}(r)$ have been calculated analytically and used as $V_{i j k l}^{\alpha \beta}$ in Eq. (1). A small difference between $w_{e}^{*}$ and $w_{h}^{*}$ is included by additional rescaling, $V_{\alpha \beta}(r)=\xi_{\alpha \beta} V(r)$, with $\xi_{\alpha \beta}^{2}=\left\langle z_{e h}^{2}\right\rangle /\left\langle z_{\alpha \beta}^{2}\right\rangle$. For $w \sim 10 \mathrm{~nm}$, we obtain $\xi_{e e}=0.94$ and $\xi_{h h}=1.08$, and for wider QW's, the difference between $w_{e}^{*}$ and $w_{h}^{*}$ is even smaller. Note that our treatment of interactions in a quasi-2D layer is different from the "biplanar" geometry (electrons and holes confined in twpparallel infinitely thin layers) tested by Chapman et al.23

The Hamiltonian $H$ is diagonalized numerically in the basis including up to five LL's $(n \leq 4)$ for both electrons and holes (note that since $l=S+n$, the inter-LL excitations of only one particle have non-zero angular momentum and, e.g., do not contribute to the $X$ ground state). Energies obtained for different values of $2 S \leq 20$ are extrapolated to $2 S \rightarrow \infty$, i.e. to an infinite QW. The eigenstates are labeled by total angular momentum $L$ and its projection $M$, which are related to the good quantum numbers on the plane: $\mathcal{L}_{\text {tot }}, \mathcal{L}_{\text {cm }}$, and $\mathcal{L}_{\text {rel }} \equiv \mathcal{L}_{\text {tot }}-\mathcal{L}_{\text {cm }}$. The total electron and hole spins $\left(J_{e}\right.$ and $\left.J_{h}\right)$ and projections $\left(J_{z e}\right.$ and $\left.J_{z h}\right)$ are also resolved.

\section{BOUND $X^{-}$STATES}

The $2 e-1 h$ energy spectra calculated for $2 S=20$ and five included electron and hole LL's $(n \leq 4)$ are shown in Fig. 1. The parameters used in the calculation $\left(w_{e}^{*}\right.$, $w_{h}^{*}$, and the dependence of $\hbar \omega_{c h}$ on $B$ ) correspond to

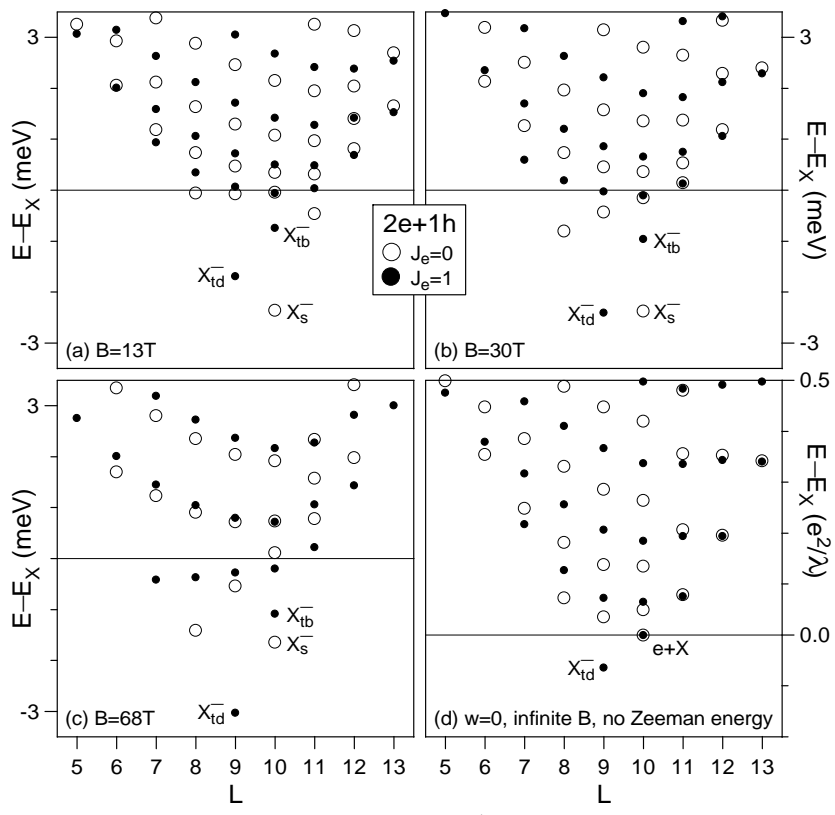

FIG. 1. The energy spectra (energy $E$ vs. angular momentum $L$ ) of the $2 e-1 h$ system on a Haldane sphere with the Landau level degeneracy of $2 S+1=21$. $E_{X}$ is the exciton energy. The parameters are appropriate for the $11.5 \mathrm{~nm}$ GaAs quantum well.

the $11.5 \mathrm{~nm}$ GaAs QW. The energy is measured from the exciton energy $E_{X}$, so that for the bound states (the states below the lines) it is opposite to the binding energy $\Delta$ (the lowest LL energy is set to zero). Open and full symbols denote singlet and triplet electron spin configurations, respectively, and only the state with the lowest Zeeman energy is marked for each triplet. Similarly, each state with $L>0$ represents a degenerate multiplet with $|M| \leq L$. The angular momentum $L$ calculated in the spherical geometry translates into angular momenta on a plane in such way 39 that the $L=S$ multiplet corresponds to $\mathcal{L}_{\text {rel }}=0$ and $\mathcal{L}_{\text {tot }}=\mathcal{L}_{\mathrm{cm}}=0,1, \ldots$, and the $L=S-1$ multiplet corresponds to $\mathcal{L}_{\text {rel }}=1$ and $\mathcal{L}_{\text {tot }}=\mathcal{L}_{\text {cm }}+1=1,2, \ldots$

Due to the conservation of $L$ in the PL process, only states from the $L=S$ channel are radiative. This is because 172526 an annihilated $e-h$ pair has $L_{X}=0$, and the final-state electron left in the lowest LL has $l_{e}=S$. Recombination of other, non-radiative $(L \neq S)$ states requires breaking rotational symmetry, e.g., by interaction with electrons, other charged complexes, or impurities. This result is independent of chosen spherical geometry and holds also for the planar QW's, where the 2D translational symmetry leads to the conservation of $\mathcal{L}_{\text {tot }}$ and $\mathcal{L}_{\mathrm{cm}}$, and the corresponding PL selection rule is $\mathcal{L}_{\text {rel }}=0$ (this simple result gan also be expressed in terms of magnetic translations 32 ).

Three states marked in Fig. 1 $1(\mathrm{a}, \mathrm{b}, \mathrm{c})(B=13,30$, and $68 \mathrm{~T})$ are of particular importance. $X_{s}^{-}$and $X_{t b}^{-}$, the lowest singlet and triplet states at $L=S$, are the only well bound radiative states, while $X_{t d}^{-}$has by far the lowest 
energy of all non-radiative $(L \neq S)$ states. The transition from the $X_{s}^{-}$to the $X_{t d}^{-}$ground state is found at $B \approx 30 \mathrm{~T}$, which confirms the calculation of WS. Our slightly larger binding energies for $w=10 \mathrm{~nm}$ are due to a larger basis used for diagonalization and including the magnetic-field dependence of the effective hole cyclotron mass (for $w \sim 10 \mathrm{~nm}, m_{h}^{*}$ increase 42 from 0.28 at $10 \mathrm{~T}$ to 0.40 at $50 \mathrm{~T}$ ). A new result is the identification of the $X_{t b}^{-}$state, which remains an excited radiative bound state in all frames (a)-(c).

For comparison, the spectrum of an ideal, strictly 2D system in the lowest LL is shown in Fig. 1(d). The $X_{t d}^{-}$is the onf bound state.20 As a result of the hidden symmetry, 14 if the only radiative states are the pair of "multiplicative" states at $L=S$ and $E=E_{X}$, in which an optically active $X$ with $L_{X}=0$ is decoupled from a free electron with $l_{e}=S$.

We have performed similar calculations for systems larger than $2 e^{-1 h}$. The results confirm that $X$ and $X^{-}$ are the only well bound $e-h$ complexes at $B \geq 10 \mathrm{~T}$. For example, the charge-neutral singlet biexciton $X_{2}$ (with $J_{e}=J_{h}=L_{X_{2}}=0$ ) unbinds at $B \approx 20 \mathrm{~T}$ even in the absence of the Zeeman splitting, and its Coulomb binding energy between 10 and $20 \mathrm{~T}$ is less than $0.1 \mathrm{meV}$.

To illustrate the finite size and surface curvature effects on the results obtained in the spherical geometry, in Fig. 2(a) we plot the Coulomb binding energies (without the Zeeman energy) of all three $X^{-}$states marked in Fig. Il(b) $(B=30 \mathrm{~T})$ as a function of $S^{-1}=(\lambda / R)^{2}$. The very regular dependence of the binding energies on the system size allows accurate extrapolation of the values obtained for $8 \leq 2 S \leq 20$ to $2 S \rightarrow \infty$, i.e. to an extended planar system $(\lambda / R=0$ and infinite LL degeneracy).

The effect of LL mixing is demonstrated in Fig. 2(b), where we plot the extrapolated binding energies $(\lambda / R=$ $0)$, calculated including between two and five electron and hole LL's, as a function of $B$. The following observations can be made. Although inclusion of one excited $(n=1)$ LL already leads to a significant $X_{s}^{-}$binding, at least the $n=2$ level must be added for the quantitatively meaningful results. Because the singlet state has more weight in the excited LL's than the triplet states, the ground-state transition shifts to higher $B$ when more LL's are included. The $X_{s}^{-}$binding energy $\Delta_{s}$ weakly depends on $B$ and saturates at $B \approx 20 \mathrm{~T}$, while $\Delta_{t d} \propto e^{2} / \lambda \propto \sqrt{B}$. Finally, the $X_{t b}^{-}$energy goes at a roughly constant separation of $1.5 \mathrm{meV}$ above $X_{s}^{-}$, and never crosses either $X_{s}^{-}$or $X_{t d}^{-}$.

To illustrate the dependence on the QW width, in Fig. $3(\mathrm{a}, \mathrm{c}, \mathrm{d})$ we compare the $X^{-}$binding energies obtained for $w=10 \mathrm{~nm}, 11.5 \mathrm{~nm}$, and $13 \mathrm{~nm}$. The thick dotted lines for $X_{s}^{-}$include the Zeeman energy needed to flip one electron's spin and form a bound spin-unpolarized state in an initially spin-polarized electron gas. The Zeeman energy $E_{Z}=g^{*} \mu_{B} B$ is roughly a linear function of energy through both cyclotron energy $\hbar \omega_{c} \propto B$ and confinement energy $\propto 1 / w^{2}$. After Snelling et al. 44 for $w \sim 10 \mathrm{~nm}$ at $B=0$, we have

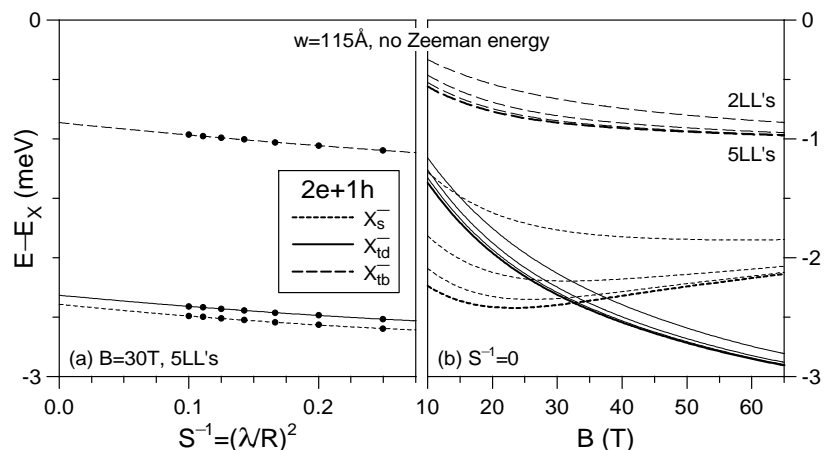

FIG. 2. (a) The $X^{-}$energies $E$ calculated on a Haldane with the Landau level (LL) degeneracy $2 S+1$, plotted as a function of $S^{-1}$, i.e. the sphere radius $R . E_{X}$ is the exciton energy and $\lambda$ is the magnetic length. Five electron and hole LL's are included. (b) The $X^{-}$binding energies $E$ extrapolated to $\lambda / R=0$, plotted as a function of the magnetic field $B$. Data obtained including different numbers of electron and hole LL's are shown; thicker lines are for five LL's. The parameters in both frames are are appropriate for the $11.5 \mathrm{~nm}$ GaAs quantum well.

$\left(g_{e}^{*}+0.29\right) w^{2}=9.4 \mathrm{~nm}^{2}$, and after Seck, Potemski, and Wyden 45 we find $d g_{e}^{*} / d B=0.0052 \mathrm{~T}^{-1}$ (for very high fields see also Ref.46). In all frames, $E_{Z}$ changes sign at $B \sim 40 \mathrm{~T}$, resulting in cusps in the $X_{s}^{-}$binding energy.

To explicitly show the magnitude of $E_{Z}$, with thin lines we also plot the $X_{s}^{-}$energy without $E_{Z}$. While the $\Delta_{s}$ including $E_{Z}$ governs the $X_{s}^{-}$relaxation and dependence of the $X_{s}^{-}$PL intensity on temperature, the $\Delta_{s}$ without $E_{Z}$ is the difference between the $X$ and $X_{s}^{-}$PL energies (neglecting the difference 3 between $g_{h}^{*}$ in the two complexes). It is clear from Fig. 3(a,c,d) that $E_{Z}$ is almost negligible for $B<50 \mathrm{~T}$ and that the binding energies are similar for all three widths.

Since only three $e-h$ complexes: $X, X_{s}^{-}$, and $X_{t b}^{-}$, have significant binding energy and at the same time belong to the radiative $L=S$ channel, only three peaks are observed in the PL spectra of dilute systems (not counting the Zeeman splittings). The total oscillator strength $\tau_{\psi}^{-1}$ of a given state $\psi$ can be expressed as

$$
\tau_{\psi}^{-1}=\left\langle\psi\left|\mathcal{P}^{\dagger} \mathcal{P}\right| \psi\right\rangle,
$$

where $\mathcal{P}^{\dagger}=\sum_{i}(-1)^{m} c_{i e}^{\dagger} c_{i h}^{\dagger}$ and $\mathcal{P}=\sum_{i}(-1)^{m} c_{i e} c_{i h}$ are the optical operators coherently creating and annihilating an $e^{-h}$ pair with $L=0$ (optically active $X$ ). In Fig. [3(b), we plot $\tau^{-1}$ of $X, X_{s}^{-}$, and $X_{t b}^{-}$as a function of $B$ for the $11.5 \mathrm{~nm}$ QW. The units of $\tau^{-1}$ follow from Eq. (3). We assume here that both electrons and holes are completely spin-polarized $\left(J_{z}=J\right)$. Typically, all electron spins and only a fraction of hole spins $\chi_{h}$ (depending on temperature and the Zeeman energy) are aligned with the field. In result, the $X_{t b}^{-}$PL has definite circular polarization $\left(\sigma_{+}\right)$and its intensity is reduced by $\chi_{h}$, while the $X_{s}^{-}$PL peak splits into a $\sigma_{ \pm}$doublet (separated by the appropriate Zeeman energy) with the intensity of the two transitions weighted by $\chi_{h}$ and $1-\chi_{h}$. 


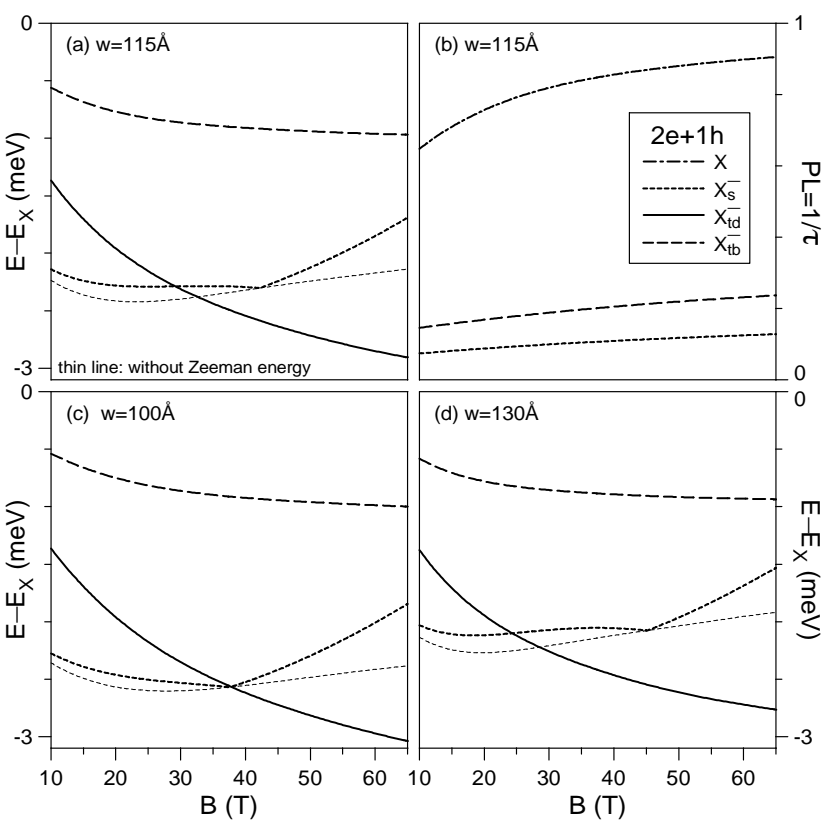

FIG. 3. The $X^{-}$binding energies $E$ (acd) and the photoluminescence intensities $\tau^{-1}$ (b) calculated for $10 \mathrm{~nm}$ (c), $11.5 \mathrm{~nm}(\mathrm{ab})$, and $13 \mathrm{~nm}$ GaAs quantum wells, plotted as a function of the magnetic field $B . E_{X}$ is the exciton energy.

In a system obeying the hidden symmetry $\left(w_{e}^{*}=w_{h}^{*}\right.$, no LL mixing, and no QW subband mixing), the total oscillator strength of one $X$ is equally shared by a pair of multiplicative $e-X$ states. In Fig. 3(b), it is distributed over a number of radiative $(L=S)$ states, and, although most is inherited by the two nearly multiplicative states at $E \approx E_{X}$, a fraction also goes to the well bound $X_{s}^{-}$ and $X_{t b}^{-}$states, with the ratio $\tau_{t b}^{-1} \approx 2 \tau_{s}^{-1}$ almost independent of $B$. The resulting three PL peaks $\left(X, X_{s}^{-}\right.$, and $\left.X_{t b}^{-}\right)$are precisely the ones observed in experiments.

The actual relative intensity of the PL peaks will depend not only on the oscillator strengths but also on the relative population of the respective initial states (i.e., efficiency of the relaxation processes, which in turn depends on the excitation energies and temperature) and their spin polarization. An increase of $\chi_{h}$ from $\frac{1}{2}$ to 1 with increasing $B$ can explain an increase of the $X_{t b}^{-} \mathrm{PL}$ intensity by up to a factor of two while the $X_{s}^{-}$PL intensity remains roughly constant.

Let us stress that the results presented in Figs. 13 are appropriate for narrow and symmetrically (or remotely) doped QW's. The agreement of the calculated binding energies and their dependence on $B$ with the experimental data for such systems 3 E is good. In much wider QW's $(w \sim 30 \mathrm{~nm})$, the subband mixing becomes significant 24 (and favors the $X_{s}^{-}$ground state), while in strongly asymmetric QW's or heterojunctions the Coulomb matrix elements $V_{i j k l}^{\alpha \beta}$ are quite different. In the latter case, the significant difference between electron and hole QW confinements $\left(w_{h}^{*} \ll w_{e}^{*}\right)$ increases the $e-h$ attraction compared to the $e-e$ repulsion within an $X^{-}$. Roughly, the binding energies of all three $X^{-}$states increase (compared to the values calculated here) by an uncompensated $e-h$ attraction which scales as $e^{2} / \lambda \propto \sqrt{B}$. This most likely explains the origin of an (equal) increase of $\Delta_{s}$ and $\Delta_{t b}$ as a function of $B$ found in Ref. 7.

While a quantitative model adequate to asymmetric QW's or heterojunctions must use correct (sampledependent) electron and hole charge density profiles $\varrho(z)$, our most important result remains valid for all structures: The triplet $X^{-}$state seen in PL is the "bright" excited triplet state at $L=S\left(\mathcal{L}_{\text {rel }}=0\right)$, while the lowest triplet state at $L=S-1\left(\mathcal{L}_{\text {rel }}=1\right)$ so far remains undetected.

It might be useful to realize which of the experimentally controlled factors generally shift the singlettriplet $X^{-}$transition to lower magnetic fields. The hidden symmetry which in Fig. 11(d) prevents binding of any other states than $X_{\text {Ed }}^{-}$is the exact overlap of electron and hole orbitals 1417 The experimentally observed binding of $X_{s}^{-}$is due to the confinement of the hole charge in a smaller volume (through asymmetric LL mixing, $\hbar \omega_{c e}>\hbar \omega_{c h}$, and asymmetric QW confinement, $w_{e}^{*}>w_{h}^{*}$ ), which enhances the $e-h$ attraction compared to the $e-e$ repulsion. Therefore, any factors should be avoided which break the $e-h$ orbital symmetry, such as (i) large $w$ leading to the QW subband mixing, (ii) well/barrier material combinations yielding $w_{h}^{*} \ll w_{h}^{*}$, (iii) large in-plane effective masses and small dielectric constants (large $\left[e^{2} / \epsilon \lambda\right] /\left[\hbar \omega_{c}\right]$ ) leading to the strong LL mixing. On the other hand, reducing $w$ strengthens Coulomb interaction and thus LL mixing, while too weak interactions (scaled e.g. by $\epsilon$ ) might decrease $X^{-}$binding energies below the experimental resolution. The giant electron Zeeman splitting $\left(g_{e}^{*} \sim 1\right)$ in CdTe or ZnSe structure 10,11 might certainly help to stabilize the $X_{t d}^{-}$ ground state at low $B$. Also, appropriate asymmetric doping producing an electric field across the QW and slightly separating electron and hole layers can help to restore balance between the $e-e$ and $e-h$ interactions.

\section{EFFECTS OF $X^{-}$INTERACTIONS}

Even in dilute systems, recombination of bound $e-h$ complexes can in principle be affected by their interaction with one another or with excess electrons. The shortrange part of the $e^{-} X^{-}$and $X^{-}-X^{-}$interaction potentials is weakened due to the $X^{-}$charge polarization, 25,26 and it is not at all obvious if even in the narrow QW's the resulting $e^{-} X^{-}$and $X^{-}-X^{-}$correlations will be similar to the Laughlin 33 correlations in an electron gas. Instead, the long-range part of the effective potentials could lead to some kind of $e^{-} X^{-}$or $X^{-}-X^{-}$pairing (in analogy to the electron or composite Fermion pairing 47 in the fractionally filled excited LL's). It has been shown 41 that the repulsion has short range and results in Laughlin correlations, if its pseudopotential $V(L)$, defined 47 as the pair interaction energy $V$ as a function of the pair angular 

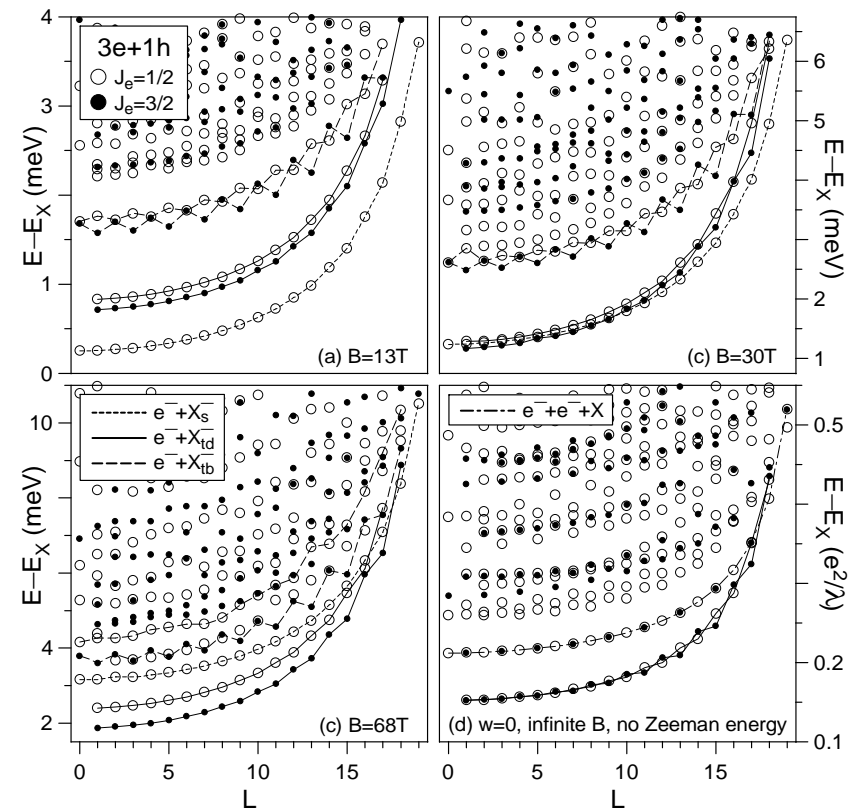

FIG. 4. The energy spectra (energy $E$ vs. angular momentum $L$ ) of the $3 e^{-1 h}$ system on a Haldane sphere with the Landau level degeneracy of $2 S+1=21$. $E_{X}$ is the exciton energy. The parameters in frames (abc) are appropriate for the $11.5 \mathrm{~nm}$ GaAs quantum well. In frame (d), $\lambda$ is the magnetic length.

momentum $L$ (on a sphere, larger $L$ means smaller separation) increases more quickly than $L(L+1)$. Therefore, the correlations in an infinite system (QW) are determined by form of the relevant pseudopotentials which can be obtained from studies of relatively small systems.

In Fig. 4 we plot the energy spectra of an $3 e-1 h$ system (the simplest system in which an $X^{-}$interacts with another charge), calculated for $2 S=20$ and three electron and hole LL's included $(n \leq 2)$. The open and filled circles mark the states with total electron spins $J_{e}=\frac{1}{2}$ and $\frac{3}{2}$, respectively, and only the lowest energy states are shown for each spin multiplet. In the low energy states, bound $X^{-}$complexes interact with an electron through the effective pseudopotentials $V(L)$, and the total energy of an interacting pair is the sum of $V(L)$ and $E_{X^{-}}$. For each pair, the allowed $L$ are obtained by adding $l_{e}=S$ of an electron and $L_{X^{-}}$of an appropriate $X^{-}$. This yields $L \geq 0$ for $X_{s}^{-}$and $X_{t b}^{-}$, and $L \geq 1$ for $X_{t d}^{-}$. However, maximum $L$ are smaller than $L_{X^{-}}+S$ due to the finite size of the $X^{-}$(hard core) 25,26 The allowed total electron spins $J_{e}$ are obtained by adding $\frac{1}{2}$ of an electron to 0 or 1 of an $X^{-}$, so that the $e^{-} X_{s}^{-}$pair states have $J_{e}=\frac{1}{2}$, while the $e-X_{t b}^{-}$and $e-X_{t d}^{-}$pair states can have either $J_{e}=\frac{1}{2}$ or $\frac{3}{2}$.

At low $L$ (i.e., at low $e-X^{-}$interaction energy compared to the $X^{-}$binding energy), the $e-X^{-}$scattering is decoupled from internal $X^{-}$dynamics, and all $e^{-} X^{-}$ pseudopotentials marked with lines in Fig. 4 are rather well approximated by those of two distinguishable point charges (electrons) with appropriate l's. Their relative position in different $e-X^{-}$bands depends on involved $\Delta$ and $E_{Z}$, and the $e-X_{t d}^{-}$states form the lowest energy band at sufficiently large $B$; see Fig. 1(c). Such regular behavior of the (two-charge) $3 e^{-1 h}$ system implies 25,26 that the lowest states of an infinite $e-h$ plasma are formed by bound $X^{-}$'s interacting with one another and with excess electrons through the Coulomb-like pseudopotentials. Depending on $B$, either $X_{s}^{-}$'s or $X_{t d}^{-}$'s form the ground state, while other bound complexes occur at higher energies, with the excitation gap given by the appropriate difference in $\Delta$ and $E_{Z}$.

Less obviously, because of the short-range 41 character of $V(L)$, the low-lying states have Laughlin-Halperin 33,34 $e-X^{-}$correlations described by a Jastrow prefactor $\prod\left(x_{i}-y_{j}\right)^{\mu}$, where $x$ and $y$ are complex coordinates of electrons and $X^{-}$'s, respectively, and $\mu$ is an integer. At fractional LL fillings $\nu=\nu_{e}-\nu_{h}, X^{-}$'s avoid41 as much as possible the $e-X^{-}$pair states with largest values of $L$. At $\nu=1 / \mu$, the ground state is the Laughlin-like incompressible fluid state with $L \leq L_{X^{-}}+S-\mu$, with quasiparticle-like excitations_described by a generalized composite Fermion model 25,26 Even though formation of an equilibrium $X^{-}$Laughlin state requires long $X^{-}$lifetime and hence is only likely for $X_{t b}^{-}$, all $X^{- \text {'s }}$ will stay as far as possible from other charges, and the distance to the nearest one corresponds to the $L=L_{X^{-}}+S-\mu$ pair state. This result depends on our assumption of the small QW width $w$. He at al.43 showed that the Laughlin $e-e$ correlations are destroyed in a thick GaAs QW when $w / \lambda>6$. At $B=40 \mathrm{~T}$, this corresponds to $w>24 \mathrm{~nm}$, but the critical width for the $e-X^{-}$correlations will be even smaller hecause of the above mentioned $X^{-}$charge polarization 2526

The connection between $\nu$ and the minimum allowed $e^{-} X^{-}$separation (or $L$ ) allows calculation of the effect of the $e-X^{-}$interaction on the $X^{-}$recombination as a function of $\nu$. In Fig. 5 we plot the PL oscillator strength $\tau^{-1}$ and energy $E$ (measured from the exciton energy $E_{X}$ ) for some of the $3 e^{-1} h$ states marked in Fig. $1(\mathrm{a}, \mathrm{b}, \mathrm{c})$. We assume that the Zeeman energy will polarize all electron spins prior to recombination, except for those two in the $X_{s}^{-}$, and concentrate on the following three initial configurations: $e^{-} X_{s}^{-}$with $J_{z e}=J_{e}=\frac{1}{2}$ and $e-X_{t b}^{-}$and $e-X_{t d}^{-}$ with $J_{z e}=J_{e}=\frac{3}{2}$. For each of the three configurations, $\tau^{-1}$ and $E$ are plotted as a function of $L$ (i.e. of $\nu$ ).

The quantities conserved in an emission process are the total angular momentum $L$ and its projection $M$ (on a plane, $\mathcal{L}_{\text {tot }}$ and $\mathcal{L}_{\mathrm{cm}}$ ), and the total electron and hole spins and their projections change by $\pm \frac{1}{2}$. For $X_{t b}^{-}$and $X_{t d}^{-}$, only an $e \uparrow-h \downarrow$ pair can be annihilated, and an emitted photon has a definite circular polarization $\sigma_{+}$. Two indistinguishable electrons left in the final state have the total spin $J_{e}=1$, so their pair angular momentum $L$ must be odd $\left(2 l_{e}\right.$ minus an odd integer). For $X_{s}^{-}$, both $\sigma_{+}$and $\sigma_{-}$PL are possible, with the energy of the latter transition shifted by the total electron and hole Zeeman energy. For $\sigma_{+}$, the two electrons in the final state can 


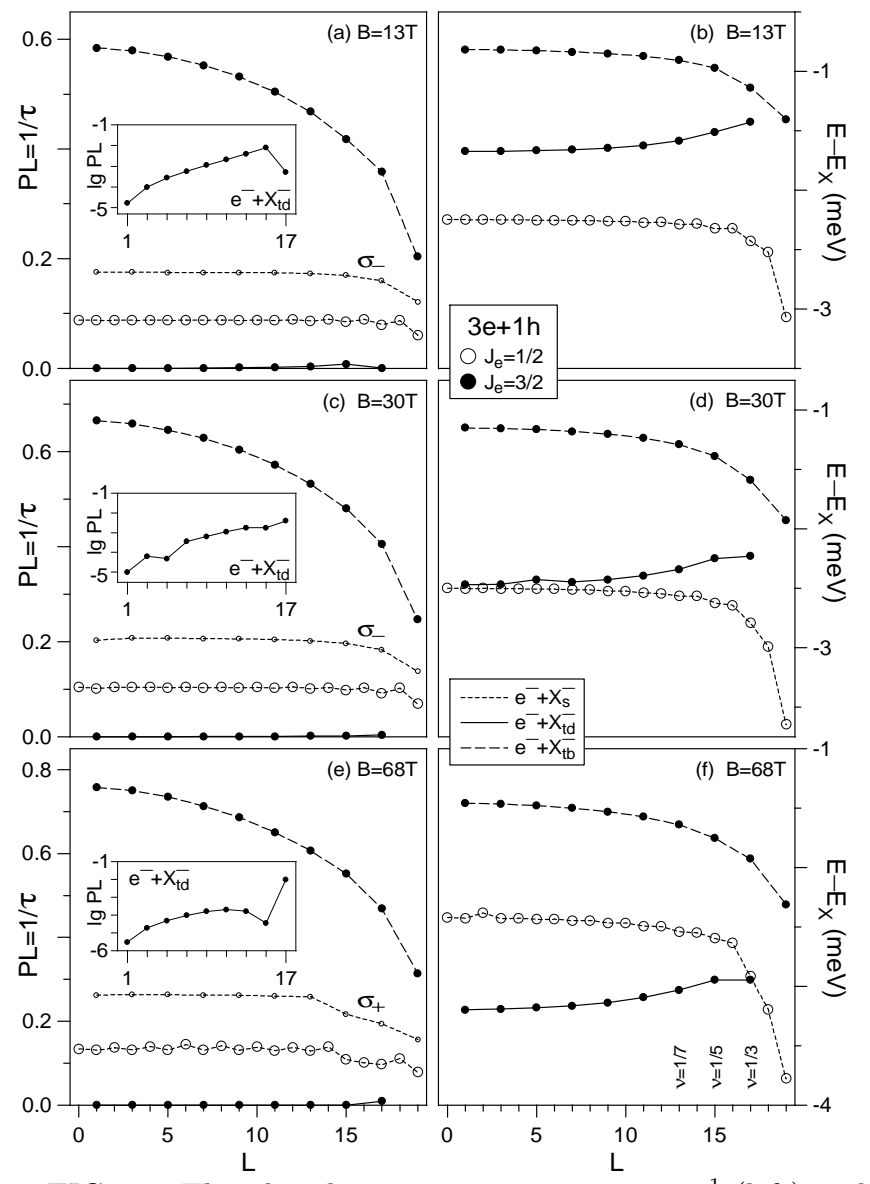

FIG. 5. The photoluminescence intensities $\tau^{-1}$ (left) and energies $E$ (right) of an $X^{-}$interacting with an electron on a Haldane sphere with the Landau level degeneracy of $2 S+1=21$, plotted as a function of the $e-X^{-}$pair angular momentum $L . E_{X}$ is the exciton energy. The parameters are appropriate for the $11.5 \mathrm{~nm}$ GaAs quantum well.

have either $J_{e}=0$ and $L$ even, or $J_{e}=1$ and $L$ odd; while for $\sigma_{+}$they can only have $J_{e}=1$ and $L$ must be odd. Note that $g_{e}^{*}$ changes sign at $B \approx 42 \mathrm{~T}$, and the polarizations in Fig. 5(e) are reversed. As expected, for $L \rightarrow 0$ the oscillator strengths converge to those of appropriate single $X^{-}$'s in Fig. B(b) (multiplied by two if only one parity of $L$ is allowed). On the right-hand side of Fig. 5, the $\sigma_{+} \mathrm{PL}$ energies are shown. For only partial polarization of hole spins, an unmarked $\sigma_{-}$peak of an $X_{s}^{-}$will appear at the energy higher by the $X^{-}$(not electron) Zeeman splitting. 5

There is no significant effect of the $e^{-} X^{-}$interactions on the $X^{-}$oscillator strength and energy at small $L$. Moreover, the decrease of the PL energy of an $X_{s}^{-}$at larger $L$ is due to its induced charge polarization (dipole moment).25.26 This effect is greatly reduced for an $X^{-}$ surrounded by an isotropic electron gas, although slight residual variation of the PL energy at $\nu \sim \frac{1}{3}$ might broaden the $X_{s}^{-}$peak. The insensitivity of the $X^{-}$recombination to the $e^{-} X^{-}$interactions at small $L$ justifies a simple picture of PL in dilute $e^{-} h$ plasmas. In this picture, recombination occurs from a single isplated bound complex and hence is virtually insensitive 6 to $\nu$. Quite surprisingly, the LH correlations prevent increase of the $X_{t d}^{-}$oscillator strength through interaction with other charges. $\tau_{t d}^{-1}$ decreases exponentially (see insets) with decreasing $\nu$, and remains ten times longer than $\tau_{s}$ even at $\nu=\frac{1}{3}$. This explains the absence of an $X_{t d}^{-}$peak even in the PL spectra 3 showing strong recombination of a higher-energy triplet state $X_{t b}^{-}$(however, see also Ref. 36).

\section{CONCLUSION}

We have studied photoluminescence (PL) from a dilute 2D electron gas in narrow and symmetric quantum wells (QW's) as a function of the magnetic field $B$ and the QW width. The puzzling qualitative discrepancy between experiments and earlier theories is resolved by identifying the radiative $\left(X_{t b}^{-}\right)$and non-radiative $\left(X_{t d}^{-}\right)$ bound states of a triplet charged exciton. Even in high magnetic fields, when it has lower energy than the radiative states, the $X_{t d}^{-}$remains invisible in PL experiments due to its negligible oscillator strength. The short range of the $e-X^{-}$interaction pseudopotentials results in the Laughlin-Halperin correlations in a dilute $e-h$ plasma, and effectively isolates the bound $X^{-}$states from the remaining electrons. This explains the observed insensitivity of the PL spectra to the filling factor and persistence of the small $X_{t d}^{-}$oscillator strength in an interacting system. An idea of the Laughlin incompressible-fluid states of long-lived $X_{t d}^{-}$'s is supported. The "dark" $X_{t d}^{-}$state could be identified either in time-resolved PL or transport experiments.

\section{ACKNOWLEDGMENT}

The authors wish to thank M. Potemski (HMFL Grenoble) and C. H. Perry and F. Munteanu (LANL Los Alamos) for helpful discussions. AW and JJQ acknowledge partial support by the Materials Research Program of Basic Energy Sciences, US Department of Energy.

${ }^{1}$ K. Kheng, R. T. Cox, Y. Merle d'Aubigne, F. Bassani, K. Saminadayar, and S. Tatarenko, Phys. Rev. Lett. 71, 1752 (1993).

${ }^{2}$ H. Buhmann, L. Mansouri, J. Wang, P. H. Beton, N. Mori, M. Heini, and M. Potemski, Phys. Rev. B 51, 7969 (1995).

${ }^{3}$ A. J. Shields, M. Pepper, M. Y. Simmons, and D. A. Ritchie, Phys. Rev. B 52, 7841 (1995); Surface Sci. 362, 451 (1996). 
${ }^{4}$ G. Finkelstein, H. Shtrikman, and I. Bar-Joseph, Phys. Rev. Lett. 74 976, (1995); Phys. Rev. B 53 R1709, (1996).

${ }^{5}$ S. Glasberg, G. Finkelstein, H. Shtrikman, and I. BarJoseph, Phys. Rev. B 59 R10 425, (1999).

${ }^{6}$ A. N. Priest, R. J. Nicholas, H. H. Cheng, M. van der Burgt, J. J. Harris, and C. T. Foxon, Physica B 249-251, 562 (1998).

${ }^{7}$ M. Hayne, C. L. Jones, R. Bogaerts, C. Riva, A. Usher, F. M. Peeters, F. Herlach, V. V. Moshchalkov, and M. Henini, Phys. Rev. B 59, 2927 (1999).

${ }^{8}$ H. A. Nickel, G. S. Herold, T. Yeo, G. Kioseoglou, Z. X. Jiang, B. D. McCombe, A. Petrou, D. Broido, and W. Schaff, Phys. Status Solidi B 210, 341 (1998).

${ }^{9}$ J. G. Tischler, B. A. Weinstein, and B. D. McCombe, Phys. Status Solidi B 215, 263 (1999).

10 T. Wojtowicz, M. Kutrowski, G. Karczewski, J. Kossut, F. J. Teran, and M. Potemski, Phys. Rev. B 59, R10 437 (1999).

${ }^{11}$ G. V. Astakhov, D. R. Yakovlev, V. P. Kochereshko, W. Ossau, J. Nürnberger, W. Faschinger, and G. Landwehr, Phys. Rev. B 60, R8485 (1999).

12 Z. X. Jiang, B. D. McCombe, and P. Hawrylak, Phys. Rev. Lett. 81, 3499 (1998).

13 S. A. Brown, J. F. Young, J. A. Brum, P. Hawrylak, and Z. Wasilewski, Phys. Rev. B 54, R11 082(1996).

14 A. H. MacDonald and E. H. Rezayi, Phys. Rev. B 42, 3224 (1990); A. H. MacDonald, E. H. Rezayi, and D. Keller, Phys. Rev. Lett. 68, 1939 (1992).

${ }^{15}$ I. V. Lerner and Yu. E. Lozovik, Zh. Eksp. Teor. Fiz. 80, 1488 (1981) [Sov. Phys. JETP 53, 763 (1981)].

16 A. B. Dzyubenko and Yu. E. Lozovik, Fiz. Tverd. Tela 25, 1519 (1983) [Sov. Phys. Solid State 25, 874 (1983)].

17 X. M. Chen and J. J. Quinn, Phys. Rev. B 50, 2354 (1994); ibid. 51, 5578 (1995).

${ }^{18}$ B. Stebe and A. Ainane, Superlatt. Microstruct. 5, 545 (1989).

19 E. I. Rashba and M. E. Portnoi, Phys. Rev. Lett. 70, 3315 (1993).

20 A. Wójs and P. Hawrylak, Phys. Rev. B 5110 880, (1995).

${ }^{21}$ J. J. Palacios, D. Yoshioka, and A. H. MacDonald, Phys. Rev. B 54, 2296 (1996).

22 A. Thilagam, Phys. Rev. B 55, 7804 (1997).

${ }^{23}$ J. R. Chapman, N. F. Johnson, and V. N. Nicopoulos, Phys. Rev. B 55, R10 221 (1997); ibid. 57, 1762 (1998).

${ }^{24}$ D. M. Whittaker and A. J. Shields, Phys. Rev. B 56, 15185 (1997).

25 A. Wójs, P. Hawrylak, and J. J. Quinn, Physica B 256258, 490 (1998); Phys. Rev. B 60, 11661 (1999).

${ }^{26}$ A. Wójs, I. Szlufarska, K. S. Yi, and J. J. Quinn, Phys. Rev. B 60, R11 273 (1999).

27 A. Moradi and B. Stebe, Solid State Commun. 112, 499 (1999).

${ }^{28}$ P. Hawrylak, Phys. Rev. B 44, 3821 (1991).

29 J. A. Brum and P. Hawrylak, Comments Cond. Matt. Phys. 18, 135 (1997).

${ }^{30}$ P. Hawrylak and M. Potemski, Phys. Rev. B 56, 12386 (1997).

31 M. A. Lampert, Phys. Rev. Lett. 1, 450 (1958).

${ }^{32}$ A. B. Dzyubenko and A. Yu. Sivachenko, unpublished (cond-mat/9902086).
33 R. B. Laughlin, Phys. Rev. Lett. 50, 1395 (1983).

${ }^{34}$ B. I. Halperin, Helv. Phys. Acta 56, 75 (1983).

${ }^{35}$ C. Lanczos, J. Res. Natn. Bur. Stand. 45, 255 (1950).

${ }^{36}$ F. M. Munteanu, Y. Kim, C. H. Perry, D. G. Rickel, J. A. Simmons, and J. L. Reno, Phys. Rev. B (to appear, Feb. 15,1999 ), observed crossing of singlet and triplet $X^{-}$states at $B \approx 40 \mathrm{~T}$. The reported triplet PL peak might be due to a collision-assisted $X_{t d}^{-}$recombination, allowed because of the lack of the LH correlations in a wider $(20 \mathrm{~nm}) \mathrm{QW}$.

${ }^{37}$ F. D. M. Haldane, Phys. Rev. Lett. 51, 605 (1983).

38 T. T. Wu and C. N. Yang, Nucl. Phys. B 107, 365 (1976).

${ }^{39}$ A. Wójs and J. J. Quinn, Physica E 3, 181 (1998).

${ }^{40}$ G. Fano, F. Orlolani, and E. Colombo, Phys. Rev. B 34, 2670 (1986).

41 A. Wójs and J. J. Quinn, Solid State Commun. 108, 493 (1998); ibid. 110, 45 (1999); Acta Phys. Pol. A 96403 (1999); unpublished (cond-mat/9903145).

42 B. E. Cole, J. M. Chamberlain, M. Henini, T. Cheng, W. Batty, A. Wittlin, J. Perenboom, A. Ardavan, A. Polisski, J. Singleton, Phys. Rev. B 55, 2503 (1997).

${ }^{43}$ S. He, F. C. Zhang, X. C. Xie, and S. Das Sarma, Phys. Rev. B 42, R11 376 (1990).

${ }^{44}$ M. J. Snelling, G. P. Flinn, A. S. Plaut, R. T. Harley, A. C. Tropper, R. Eccleston, and C. C. Philips, Phys. Rev. B 44, 11345 (1991).

${ }^{45}$ M. Seck, M. Potemski, and P. Wyder, Phys. Rev. B 56, 7422 (1997).

${ }^{46}$ S. P. Najda, S. Takejama, N. Miura, P. Pfeffer, and W. Zawadzki, Phys. Rev. B 40, 6189 (1989).

${ }^{47}$ F. D. M. Haldane and E. H. Rezayi, Phys. Rev. Lett. 60, 956 (1988). 